\title{
Global Asymptotic Almost Periodic Synchronization of Clifford-Valued CNNs with Discrete Delays
}

\author{
Yongkun Li (iD) and Jianglian Xiang \\ Department of Mathematics, Yunnan University, Kunming, Yunnan 650091, China \\ Correspondence should be addressed to Yongkun Li; yklie@ynu.edu.cn
}

Received 14 February 2019; Accepted 18 April 2019; Published 12 May 2019

Academic Editor: Xinzhi Liu

Copyright (c) 2019 Yongkun Li and Jianglian Xiang. This is an open access article distributed under the Creative Commons Attribution License, which permits unrestricted use, distribution, and reproduction in any medium, provided the original work is properly cited.

\begin{abstract}
In this paper, we are concerned with Clifford-valued cellular neural networks (CNNs) with discrete delays. Since Clifford algebra is a unital associative algebra and its multiplication is noncommutative, to overcome the difficulty of the noncommutativity of the multiplication of Clifford numbers, we first decompose the considered Clifford-valued neural network into $2^{m^{2}} n$ real-valued systems. Second, based on the Banach fixed point theorem, we establish the existence and uniqueness of almost periodic solutions of the considered neural networks. Then, by designing a novel state-feedback controller and constructing a proper Lyapunov function, we study the global asymptotic synchronization of the considered neural networks. Finally, a numerical example is presented to show the effectiveness and feasibility of our results.
\end{abstract}

\section{Introduction}

Clifford algebras, which were invented by mathematician W. K. Clifford, are algebras generated from vector spaces with quadratic forms and are unitary associative algebras. They contain real numbers, complex numbers, quaternions, and several other hypercomplex systems as special cases. Clifford algebras have important applications in a variety of fields, including geometry, theoretical physics, and digital image processing [1]. Clifford-valued neural networks generalize real-valued, complex-valued, quaternion-valued, and octonion-valued neural networks and so on. Since the Clifford-valued neural networks can use multistate activation functions to process multilevel information and require much fewer connection weight parameters of the networks, they have recently been an active research area [2-5]. Due to the noncommutativity of Clifford numbers' multiplication, it brings great difficulties to the research of Clifford-valued neural networks. At present, the results of research on Clifford-valued neural networks are still rare, especially, for the nonautonomous ones.

On the one hand, it is well known that, in the design and implementation of neural networks, the existence of periodic solutions or almost periodic solutions of nonautonomous neural networks is as important as the existence of equilibrium points of autonomous neural networks. At the same time, we know that, even if all the time-varying coefficients in a neural network are periodic functions (such as connection coefficients, connection weight functions, external inputs, and time delays, etc.), if their periods are incommensurable, then, for such a neural network, it is also impossible to have a periodic solution. Therefore, studying the almost periodicity of neural networks is more practical and important than studying the periodicity of neural networks. At present, the periodicity has been extensively studied as an important dynamic property of various neural networks. However, there has been no paper published on the almost periodicity of Clifford-valued neural networks yet.

On the other hand, the synchronization of nonlinear systems has become an important research topic due to its potential applications in various fields such as secure communication, image encryption, information science, and so on. Particularly, recently, many authors have studied the synchronization problem for various neural network systems [6-15]. For example, the synchronization problem for chaotic memristor-based neural networks with time-varying delays 
was studied in [11]. The global asymptotic synchronization problem of nonidentical fractional-order neural networks with Riemann-Liouville derivative was investigated in [12]. The synchronization of an inertial neural network with time-varying delays was investigated in [13]. The finitetime cluster synchronization of coupled fuzzy cellular neural networks with Markovian switching topology, discontinuous activation functions, proportional leakage, and time-varying unbounded delays was studied in [14]. The global exponential almost periodic synchronization of quaternion-valued neural networks with time-varying delays was investigated in [15]. But until now, the results of the synchronization of Cliffordvalued neural networks have not been reported.

In summary, it is meaningful to study the almost periodicity and the synchronization problem of Clifford-valued neural networks. Therefore, our main purpose of this paper is to investigate the problem of the existence of almost periodic solutions and global asymptotic synchronization of Clifford-valued CNNs with discrete delays. To the best of our knowledge, this is the first paper to study such a problem for Clifford-valued neural networks with discrete delays. Our methods of this paper can be used to study other types of Clifford-valued neural networks.

The rest of this paper is organized as follows: in Section 2, we introduce some basic concepts, notations, and lemmas and give a model description. In Section 3, we study the existence of almost periodic solutions of Clifford-valued CNNs with discrete delays. In Section 4, we investigate the global asymptotic almost periodic synchronization of Clifford-valued CNNs with discrete delays. In Section 5, we give an example to demonstrate the effectiveness and feasibility of our results. In Section 5, we give a conclusion.

\section{Preliminaries and Model Description}

The real Clifford algebra over $\mathbb{R}^{m}$ is defined as

$$
\mathscr{A}=\left\{\sum_{A \subseteq\{1,2, \ldots, m\}} a_{A} e_{A}, a_{A} \in \mathbb{R}\right\},
$$

where $e_{A}=e_{h_{1}} e_{h_{2}} \cdots e_{h_{\nu}}$ with $A=\left\{h_{1}, h_{2}, \ldots, h_{\gamma}\right\}, 1 \leq h_{1}<$ $h_{2}<\cdots<h_{v} \leq m$. Moreover, $e_{\emptyset}=e_{0}=1$ and $e_{\{h\}}=$ $e_{h}, h=1,2, \ldots, m$ are called Clifford generators which satisfy the relations $e_{i}^{2}=-1, i=1,2, \ldots, m$, and $e_{i} e_{j}+e_{j} e_{i}=0, i \neq$ $j, i, j=1,2, \ldots, m$.

For simplicity, when one element is the product of multiple Clifford generators, we will write its subscripts together. For example, $e_{4} e_{5} e_{7} e_{9}=e_{4579}$. Denote $\Lambda=$ $\{\emptyset, 1,2, \ldots, A, \ldots, 12 \cdots m\}$, and then we have

$$
\mathscr{A}=\left\{\sum_{A} a_{A} e_{A}, a_{A} \in \mathbb{R}\right\},
$$

where $\sum_{A}$ is short for $\sum_{A \in \Lambda}$ and $\mathscr{A}$ is isomorphic to $\mathbb{R}^{2^{m}}$.

For any $x=\sum_{A} x_{A} \in \mathscr{A}$, the involution of $x$ is defined as

$$
\bar{x}=\sum_{A} x_{A} \bar{e}_{A},
$$

where $\bar{e}_{A}=(-1)^{\mu[A](\mu[A]+1) / 2} e_{A}$, and if $A=\emptyset$, then $\mu[A]=0$; if $A=h_{1} h_{2} \ldots h_{v} \in \Lambda$, then $\mu[A]=\nu$. It is easy to see that $e_{A} \bar{e}_{A}=\bar{e}_{A} e_{A}=1$ and for every $x=\sum_{A} x^{A} e_{A} \in \mathscr{A}, \bar{x}=$ $\sum_{A} x^{A} \bar{e}_{A}$. Moreover, we have $\overline{x y}=\bar{y} \bar{x}, \forall x, y \in \mathscr{A}$.

For a Clifford-valued function $z=\sum_{A} z^{A} e_{A}: \mathbb{R} \longrightarrow \mathscr{A}$, where $z^{A}: \mathbb{R} \longrightarrow \mathbb{R}, A \in \Lambda$, its derivative is given by

$$
\dot{z}(t)=\sum_{A} \dot{z}^{A}(t) e_{A}
$$

In view of $e_{B} \bar{e}_{A}=(-1)^{\mu[A](\mu[A]+1) / 2} e_{B} e_{A}$, we can write $e_{B} \bar{e}_{A}=$ $e_{C}$ or $e_{B} \bar{e}_{A}=-e_{C}$, where $e_{C}$ is a Clifford generator of Clifford algebra $\mathscr{A}$. Hence it is possible to find a unique corresponding basis $e_{C}$ for the given $e_{B} \bar{e}_{A}$. Define

$$
\mu[B \cdot \bar{A}]= \begin{cases}0, & \text { if } e_{B} \bar{e}_{A}=e_{C} \\ 1, & \text { if } e_{B} \bar{e}_{A}=-e_{C}\end{cases}
$$

and then $e_{B} \bar{e}_{\mathrm{A}}=(-1)^{\mu[B \cdot \bar{A}]} e_{C}$. In addition, for any $E \in \mathscr{A}$, we can find a unique $E^{C}$ satisfying $E^{B \cdot \bar{A}}=(-1)^{\mu[B \cdot \bar{A}]} E^{C}$ for $e_{B} \bar{e}_{A}=(-1)^{\mu[B \cdot \bar{A}]} e_{C}$. Therefore,

$$
\begin{aligned}
E^{B \cdot \bar{A}} e_{B} \bar{e}_{A} & =E^{B \cdot \bar{A}}(-1)^{\mu[B \cdot \bar{A}]} e_{C} \\
& =(-1)^{\mu[B \cdot \bar{A}]} E^{C}(-1)^{\mu[B \cdot \bar{A}]} e_{C}=E^{C} e_{C}
\end{aligned}
$$

and

$$
E=\sum_{C} E^{C} e_{C} \in \mathscr{A}
$$

Throughout this paper, $\mathscr{A}^{n}, \mathbb{R}^{m \times n}, \mathscr{A}^{m \times n}$ represent the $n$ dimensional real Clifford vector space, the set of all $m \times n$ real matrices, and the set of all $m \times n$ real Clifford matrices. We define the norm of $\mathbb{R}^{n}$ as $\|x\|=\sum_{i=1}^{n}\left|x_{i}\right|$. For $A=\left(a_{i j}\right)_{n \times n}$, denote $\|A\|=\max _{1 \leq i \leq n}\left\{\sum_{j=1}^{n}\left|a_{i j}\right|\right\}$ and, for $x=\sum_{A} x^{A} e_{A} \in \mathscr{A}$, denote $\|x\|_{\mathscr{A}}=\sum_{A}\left|x^{A}\right|$.

In this paper, we consider the following Clifford-valued cellular neural network with discrete delays:

$$
\begin{aligned}
\dot{X}_{i}(t)= & -\delta_{i}(t) X_{i}(t)+\sum_{j=1}^{n} \gamma_{i j}(t) f_{j}\left(X_{j}(t)\right) \\
& +\sum_{j=1}^{n} \omega_{i j}(t) g_{j}\left(X_{j}\left(t-\tau_{j}(t)\right)\right)+I_{i}(t),
\end{aligned}
$$

$$
t>0
$$

where $i=1,2, \ldots, n$, and $n$ corresponds to the number of units in the neural network; $X_{i}(t) \in \mathscr{A}$ denotes the activation of the $i$ th neuron at time $t ; \delta_{i}(t)>0$ represents the rate with which the $i$ th unit will reset its potential to the resting state in isolation when disconnected from the network and external inputs at time $t ; \gamma_{i j}(t), \omega_{i j}(t) \in \mathscr{A}$ represent the strengths of connectivity without and with time delay between cells $i$ and $j$ at time $t$, respectively; $f_{j}, g_{j}: \mathscr{A} \longrightarrow \mathscr{A}$ are the activation functions of the signal transmission; $I_{i}(t) \in \mathscr{A}$ is an external 
input on the $i$ th unit at time $t ; \tau_{j}(t) \geq 0$ is the transmission delay at time $t$.

The initial values of (8) are

$$
X_{i}(t)=\varphi_{i}(t), \quad t \in[-\tau, 0], i=1,2, \ldots, n,
$$

where $\varphi_{i} \in C([-\tau, 0], \mathscr{A}), \tau=\max _{\{1 \leq j \leq n\}} \sup _{t \in \mathbb{R}}\left\{\left|\tau_{j}(t)\right|\right\}$.

Let $X(t)=\left(X_{1}(t), X_{2}(t), \ldots, X_{n}(t)\right)^{T} \in \mathscr{A}^{n}, \Delta(t)=$ $\operatorname{diag}\left(\delta_{1}(t), \delta_{2}(t), \ldots, \delta_{n}(t)\right) \in \mathbb{R}^{n \times n}, \Gamma(t)=\left(\gamma_{i j}(t)\right)_{n \times n}, \Omega(t)=$ $\left(\omega_{i j}(t)\right)_{n \times n} \in \mathscr{A}^{n \times n}, I(t)=\left(I_{1}(t), I_{2}(t), \ldots, I_{n}(t)\right)^{T} \in \mathscr{A}^{n}$, $f(X(t))=\left(f_{1}\left(X_{1}(t)\right), f_{2}\left(X_{2}(t)\right), \ldots, f_{n}\left(X_{n}(t)\right)\right)^{T} \in \mathscr{A}^{n}$, and $g(X(t-\tau(t)))=\left(g_{1}\left(X_{1}\left(t-\tau_{1}(t)\right)\right), g_{2}\left(X_{2}\left(t-\tau_{2}(t)\right)\right), \ldots\right.$, $\left.g_{n}\left(X_{n}\left(t-\tau_{n}(t)\right)\right)\right)^{T} \in \mathscr{A}^{n}$. Then (8) can be transformed into the following equation:

$$
\begin{aligned}
\dot{X}(t)= & -\Delta(t) X(t)+\Gamma(t) f(X(t)) \\
& +\Omega(t) g(X(t-\tau(t)))+I(t), \quad t>0
\end{aligned}
$$

with the initial value:

$$
\begin{aligned}
X(t) & =\varphi(t), \\
& t \in[-\tau, 0], \varphi(t)=\left(\varphi_{1}(t), \varphi_{2}(t), \ldots, \varphi_{n}(t)\right)^{T} .
\end{aligned}
$$

Definition 1 (see [16]). A function $y \in C\left(\mathbb{R}, \mathbb{R}^{n}\right)$ is said to be almost periodic on $\mathbb{R}$, if, for any $\varepsilon>0$, it is possible to find a real number $l=l(\varepsilon)>0$ such that, for any interval with length $l(\varepsilon)$, there is a number $\tau=\tau(\varepsilon)$ in this interval such that $|y(t+\tau)-y(t)|<\varepsilon$.

Denote by $A P\left(\mathbb{R}, \mathbb{R}^{n}\right)$ the set of all almost periodic functions on $\mathbb{R}$; then $A P\left(\mathbb{R}, \mathbb{R}^{n}\right)$ is a Banach space with the supremum norm $\|u\|=\sum_{i=1}^{n} \sup _{t \in \mathbb{R}}\left|u_{i}(t)\right|$.

Definition 2. A function $f=\left(f_{1}, f_{2}, \ldots, f_{n}\right)^{T}: \mathbb{R} \longrightarrow \mathscr{A}^{n}$, where $f_{i}=\sum_{A} f_{i}^{A} e_{A}, f_{i}^{A}: \mathbb{R} \longrightarrow \mathbb{R}$, is called almost periodic if every $f_{i}^{A} \in A P(\mathbb{R}, \mathbb{R}), i=1,2, \ldots, n$. Denote by $A P\left(\mathbb{R}, \mathscr{A}^{n}\right)$ the set of all such functions.

Consider the following linear system

$$
\dot{x}(t)=Q(t) x(t)+f(t),
$$

where $Q(t)=\operatorname{diag}\left\{-q_{1}(t),-q_{2}(t), \ldots, q_{n}(t)\right\}, q_{i} \quad \epsilon$ $A P(\mathbb{R}, \mathbb{R}), f \in A P\left(\mathbb{R}, \mathbb{R}^{n}\right)$.

According to [16], one can easily get the following lemma.

Lemma 3. Let $q_{i}(t)$ satisfy $\min _{1 \leq i \leq n} \inf _{t \in \mathbb{R}}\left\{q_{i}(t)\right\}>0$, and then system (12) has a unique almost periodic solution

$$
x(t)=\int_{-\infty}^{t} e^{\int_{s}^{t} Q(u) d u} f(s) d s
$$

\section{The Existence of Almost Periodic Solutions}

In this section, we study the existence and uniqueness of almost periodic solutions by the contracting mapping principle.
Firstly, since, for any $W \in \mathscr{A}$, we can find a unique $W^{C}$ satisfying $W^{B \cdot \bar{A}}=(-1)^{n(B \cdot \bar{A})} W^{C}$ for $e_{B} \bar{e}_{A}=(-1)^{n(B \cdot \bar{A})} e_{C}$, then, based on $e_{A} \bar{e}_{A}=\bar{e}_{A} e_{A}=1$ and $e_{B} \bar{e}_{A} e_{A}=e_{B}$, we can transform Clifford-valued system (10) into the following realvalued neural network:

$$
\begin{aligned}
\dot{x}^{A}(t)= & -\Delta(t) x^{A}(t)+\sum_{B} \Gamma^{A \cdot \bar{B}}(t) f^{B}(x(t)) \\
& +\sum_{B} \Omega^{A \cdot \bar{B}}(t) g^{B}\left(x^{\tau}(t)\right)+I^{A}(t), \quad A \in \Lambda,
\end{aligned}
$$

where

$$
\begin{aligned}
& x^{A}=\left(x_{1}^{A}, x_{2}^{A}, \ldots, x_{n}^{A}\right)^{T}, \\
& \sum_{A} x^{A} e_{A}=X \\
& (-1)^{n(A \cdot \bar{B})} \Gamma^{C}=\Gamma^{A \cdot \bar{B}}, \\
& (-1)^{n(A \cdot \bar{B})} \Omega^{C}=\Omega^{A \cdot \bar{B}}, \\
& \sum_{C} \Gamma^{C} e_{C}=\Gamma, \\
& \sum_{C} \Omega^{C} e_{C}=\Omega, \\
& e_{A} \overline{e_{B}}=(-1)^{n(A \cdot \bar{B})} e_{C}, \\
& \Gamma^{A \cdot \bar{B}}=\left(\gamma_{i j}^{A \cdot \bar{B}}\right)_{n \times n}, \\
& \Omega^{A \cdot \bar{B}}=\left(\omega_{i j}^{A \cdot \bar{B}}\right)_{n \times n}, \\
& I^{A}=\left(I_{1}^{A}, I_{2}^{A}, \ldots, I_{n}^{A}\right)^{T}, \\
& f^{B}(x)=\left(f_{1}^{B}\left(x_{1}^{C_{1}}, x_{1}^{C_{2}}, \ldots, x_{1}^{C_{2} m}\right),\right. \\
& f_{2}^{B}\left(x_{2}^{C_{1}}, x_{2}^{C_{2}}, \ldots, x_{2}^{C_{2} m}\right), \ldots, \\
& \left.f_{n}^{B}\left(x_{n}^{C_{1}}, x_{n}^{C_{2}}, \ldots, x_{n}^{C_{2} m}\right)\right)^{T}, \\
& g^{B}\left(x^{\tau}(t)\right)=\left(g _ { 1 } ^ { B } \left(x_{1}^{C_{1}}\left(t-\tau_{1}(t)\right), x_{1}^{C_{2}}\left(t-\tau_{1}(t)\right), \ldots,\right.\right. \\
& \left.x_{1}^{C_{2} m}\left(t-\tau_{1}(t)\right)\right), g_{2}^{B}\left(x_{2}^{C_{1}}\left(t-\tau_{2}(t)\right), x_{2}^{C_{2}}\left(t-\tau_{2}(t)\right),\right. \\
& \left.\ldots, x_{2}^{C_{2} m}\left(t-\tau_{2}(t)\right)\right), \ldots, g_{n}^{B}\left(x_{n}^{C_{1}}\left(t-\tau_{n}(t)\right),\right. \\
& \left.x_{n}^{C_{2}}\left(t-\tau_{n}(t)\right), \ldots, x_{n}^{C_{2^{m}}}\left(t-\tau_{n}(t)\right)\right)^{T} \text {. }
\end{aligned}
$$

Remark 4. If $x(t)=\left(x^{0}(t), x^{1}(t), \ldots, x^{A}(t), \ldots, x^{1 \cdot 2 \cdots \cdot m}(t)\right)^{T}$ is a solution of (14), then $X(t)=\left(X_{1}, X_{2}, \ldots, X_{n}\right)^{T}=$ $\left(\sum_{A} x_{1}^{A}(t) e_{A}, \sum_{A} x_{2}^{A}(t) e_{A}, \ldots, \sum_{A} x_{n}^{A}(t) e_{A}\right)^{T}$ is a solution of (8) and vice versa. 


\section{Denote}

$$
\begin{aligned}
& S=\left(\left(x^{0}\right)^{T},\left(x^{1}\right)^{T},\left(x^{2}\right)^{T}, \ldots,\left(x^{A}\right)^{T}, \ldots,\left(x^{12 \ldots m}\right)^{T}\right)^{T} \in \mathbb{R}^{2^{m} n} \\
& \tilde{f}(S)=\left(\left(f^{0}(x)\right)^{T},\left(f^{1}(x)\right)^{T},\left(f^{2}(x)\right)^{T}, \ldots,\left(f^{A}(x)\right)^{T}, \ldots,\left(f^{12 \ldots m}(x)\right)^{T}\right)^{T}, \\
& \tilde{g}\left(S^{\tau}\right)=\left(\left(g^{0}\left(x^{\tau}\right)\right)^{T},\left(g^{1}\left(x^{\tau}\right)\right)^{T},\left(g^{2}\left(x^{\tau}\right)\right)^{T}, \ldots,\left(g^{A}\left(x^{\tau}\right)\right)^{T}, \ldots,\left(g^{12 \ldots m}\left(x^{\tau}\right)\right)^{T}\right)^{T}, \\
& \widetilde{I}(t)=\left(\left(I^{0}\right)^{T},\left(I^{1}\right)^{T},\left(I^{2}\right)^{T}, \ldots,\left(I^{A}\right)^{T}, \ldots,\left(I^{12 \ldots m}\right)^{T}\right)^{T}, \\
& \widetilde{\Delta}=\left(\begin{array}{cccc}
\Delta & 0 & \cdots & 0 \\
0 & \Delta & \cdots & 0 \\
\vdots & \vdots & \ddots & \vdots \\
0 & 0 & \cdots & \Delta
\end{array}\right)_{2^{m} n \times 2^{m} n} \\
& \widetilde{\Gamma}=\left(\begin{array}{ccccc}
\Gamma^{0} & \ldots & \Gamma^{\bar{A}} & \cdots & \Gamma^{\overline{12 \ldots m}} \\
\Gamma^{1} & \cdots & \Gamma^{1 \cdot \bar{A}} & \cdots & \Gamma^{1 \cdot \overline{12 \ldots m}} \\
\vdots & \ldots & \vdots & \ldots & \vdots \\
\Gamma^{12 \ldots m} & \ldots & \Gamma^{12 \ldots m \cdot \bar{A}} & \cdots & \Gamma^{12 \ldots m \cdot \overline{12 \ldots m}}
\end{array}\right)_{2^{m} n \times 2^{m} n}, \\
& \widetilde{\Omega}=\left(\begin{array}{ccccc}
\Omega^{0} & \ldots & \Omega^{\bar{A}} & \cdots & \Omega^{\overline{12 \ldots m}} \\
\Omega^{1} & \cdots & \Omega^{1 \cdot \bar{A}} & \ldots & \Omega^{1 \cdot \overline{12 \ldots m}} \\
\vdots & \ldots & \vdots & \cdots & \vdots \\
\Omega^{12 \ldots m} & \ldots & \Omega^{12 \ldots m \cdot \bar{A}} & \cdots & \Omega^{12 \ldots m \cdot \overline{12 \ldots m}}
\end{array}\right)_{2^{m} n \times 2^{m} n},
\end{aligned}
$$

and then (14) can be written as

$$
\begin{aligned}
\dot{S}(t)= & -\widetilde{\Delta}(t) S(t)+\widetilde{\Gamma}(t) \tilde{f}(S(t))+\widetilde{\Omega}(t) \widetilde{g}\left(S^{\tau}(t)\right) \\
& +\widetilde{I}(t), \quad t>0
\end{aligned}
$$

with the initial value:

$$
S(t)=\phi(t), \quad t \in[-\tau, 0] .
$$

Set $\mathbb{X}=\left\{\Phi \mid \Phi=\left(\left(\Phi^{0}\right)^{T},\left(\Phi^{1}\right)^{T}, \ldots,\left(\Phi^{1 \cdot 2 \cdots m}\right)^{T}\right)^{T} \in\right.$ $\left.A P\left(\mathbb{R}, \mathbb{R}^{2^{m} n}\right)\right\}$ with the norm

$$
\begin{aligned}
\|\Phi\|_{0}= & \sum_{i=1}^{n} \sum_{A}\left|\Phi_{i}^{A}\right|, \\
\left|\Phi_{i}^{A}\right|=\sup _{t \in R}\left|\Phi_{i}^{A}(t)\right|, & \\
& i=1,2, \ldots, n,
\end{aligned}
$$

which is a Banach space.

In order to obtain our results, we introduce the following assumptions.
$\left(H_{1}\right)$ For $i, j=1,2, \ldots, n, \delta_{i}, \tau_{j} \in A P\left(\mathbb{R}, \mathbb{R}^{+}\right), \gamma_{i j}, \omega_{i j}, I_{i j} \in$ $A P(\mathbb{R}, \mathscr{A})$ and for each $1 \leq i \leq n$, $\min _{1 \leq i \leq n}\left\{\inf _{t \in \mathbb{R}} c_{i}(t)\right\}>0$.

$\left(H_{2}\right)$ For $i=1,2, \ldots, n, f_{i}, g_{i} \in C(\mathscr{A}, \mathscr{A})$ and there exist constants $L_{i}^{f}, L_{i}^{g}$ such that

$$
\begin{aligned}
& \left\|f_{i}(x)-f_{i}(y)\right\|_{\mathscr{A}} \leq L_{i}^{f}\|x-y\|_{\mathscr{A}}, \\
& \left\|g_{i}(x)-g_{i}(y)\right\|_{\mathscr{A}} \leq L_{i}^{g}\|x-y\|_{\mathscr{A}},
\end{aligned}
$$

and there exist constants $F, G>0$ such that $\|f(x)\|_{\mathscr{A}} \leq$ $F,\|g(x)\|_{\mathscr{A}} \leq G$, for any $x, y \in \mathscr{A}$.

Remark 5. By $\left(\mathrm{H}_{2}\right)$, we can obtain

$$
\begin{aligned}
& \sum_{A}\left|f_{i}^{A}\left(y_{i}^{B_{1}}, y_{i}^{B_{2}}, \ldots, y_{i}^{B_{2} m}\right)-f_{i}^{A}\left(x_{i}^{B_{1}}, x_{i}^{B_{2}}, \ldots, x_{i}^{B_{2} m}\right)\right| \\
& \quad \leq L_{i}^{f} \sum_{B}\left|y_{i}^{B}-x_{i}^{B}\right|
\end{aligned}
$$




$$
\begin{aligned}
& \sum_{A}\left|g_{i}^{A}\left(y_{i}^{B_{1}}, y_{i}^{B_{2}}, \ldots, y_{i}^{B_{2} m}\right)-g_{i}^{A}\left(x_{i}^{B_{1}}, x_{i}^{B_{2}}, \ldots, x_{i}^{B_{2} m}\right)\right| \\
& \quad \leq L_{i}^{g} \sum_{B}\left|y_{i}^{B}-x_{i}^{B}\right| .
\end{aligned}
$$

Theorem 6. Let $\left(H_{1}\right)$ and $\left(H_{2}\right)$ hold. Suppose that

$$
\begin{aligned}
\left(H_{3}\right)\left(\widetilde{\Gamma}^{+} L_{f}+\bar{L}^{+} L_{g}\right)\left(1 / \widetilde{\Delta}^{-}\right)<1, \text { where } \\
\widetilde{\Gamma}^{+}=\sup _{t \in \mathbb{R}}\|\widetilde{\Gamma}(t)\|, \\
\widetilde{\Omega}^{+}=\sup _{t \in \mathbb{R}}\|\widetilde{\Omega}(t)\|, \\
\widetilde{\Delta}^{-}=\inf _{t \in \mathbb{R}}\|\widetilde{\Delta}(t)\|, \\
\widetilde{I}^{+}=\sup _{t \in \mathbb{R}}\|\widetilde{I}(t)\|, \\
L_{f}=\max _{1 \leq i \leq n}\left\{L_{i}^{f}\right\}, \\
L_{g}=\max _{1 \leq i \leq n}\left\{L_{i}^{g}\right\} .
\end{aligned}
$$

Then system of (8) has a unique almost periodic solution in the region

$$
\begin{aligned}
\mathbb{X}_{0} & \\
& =\left\{\Phi \in \mathbb{X} \mid\|\Phi\|_{0} \leq\left(\widetilde{\Gamma}^{+} F+\widetilde{\Omega}^{+} G+\widetilde{I}^{+}\right) \frac{1}{\widetilde{\Delta}^{-}}:=R\right\} .
\end{aligned}
$$

Proof. For $\forall \Phi \in \mathbb{X}$, we consider the following system:

$$
\begin{aligned}
\dot{S}(t)= & -\widetilde{\Delta}(t) S(t)+\widetilde{\Gamma}(t) \widetilde{f}(\Phi(t)) \\
& +\widetilde{\Omega}(t) \widetilde{g}(\Phi(t-\tau(t)))+\widetilde{I}(t) .
\end{aligned}
$$

Since $\left(H_{1}\right)$, it follows from Lemma 3 that system $(24)$ has a unique almost periodic solution

$$
\begin{aligned}
& S(t)=\int_{-\infty}^{t} e^{\int_{s}^{t}-\widetilde{\Delta}(u) d u}[\widetilde{\Gamma}(s) \widetilde{f}(\Phi(s)) \\
&+\widetilde{\Omega}(s) \widetilde{g}(\Phi(s-\tau(s)))+\widetilde{I}(s)] d s .
\end{aligned}
$$

We define a mapping $T: \mathbb{X}_{0} \longrightarrow \mathbb{X}$ as follows:

$$
\begin{gathered}
(T \Phi)(t)=\int_{-\infty}^{t} e^{\int_{s}^{t}-\widetilde{\Delta}(u) d u}[\widetilde{\Gamma}(s) \widetilde{f}(\Phi(s)) \\
+\widetilde{\Omega}(s) \widetilde{g}(\Phi(s-\tau(s)))+\widetilde{I}(s)] d s .
\end{gathered}
$$

We first prove that $T\left(\mathbb{X}_{0}\right) \subset \mathbb{X}_{0}$. To this end, for each $\Phi \epsilon$ $\mathbb{X}_{0}$, we have

$$
\begin{aligned}
& \|(T \Phi)(t)\|=\| \int_{-\infty}^{t} e^{\int_{s}^{t}-\widetilde{\Delta}(u) d u}[\widetilde{\Gamma}(s) \tilde{f}(\Phi(s)) \\
& +\widetilde{\Omega}(s) \widetilde{g}(\Phi(s-\tau(s)))+\widetilde{I}(s)] d s \| \\
& \leq \int_{-\infty}^{t}\left\|e^{\int_{s}^{t}-\widetilde{\Delta}(u) d u}\right\|\|\widetilde{\Gamma}(s)\|\|\widetilde{f}(\Phi(s))\| d s
\end{aligned}
$$

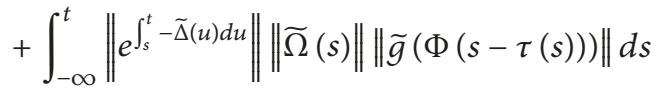

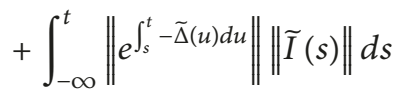

$$
\begin{aligned}
& \leq \int_{-\infty}^{t} e^{-\widetilde{\Delta}^{-}(t-s)} \widetilde{\Gamma}^{+}\|\tilde{f}(\Phi(s))\| d s \\
& +\int_{-\infty}^{t} e^{-\widetilde{\Delta}^{-}(t-s)} \widetilde{\Omega}^{+}\|\widetilde{g}(\Phi(s-\tau(s)))\| d s \\
& +\int_{-\infty}^{t} e^{-\widetilde{\Delta}^{-}(t-s)} \widetilde{I}^{+} d s \leq \widetilde{\Gamma}^{+} F \int_{-\infty}^{t} e^{-\widetilde{\Delta}^{-}(t-s)} d s \\
& +\widetilde{\Omega}^{+} G \int_{-\infty}^{t} e^{-\widetilde{\Delta}^{-}(t-s)} d s+\widetilde{I}^{+} \int_{-\infty}^{t} e^{-\widetilde{\Delta}^{-}(t-s)} d s \\
& \leq\left(\widetilde{\Gamma}^{+} F+\widetilde{\Omega}^{+} G+\widetilde{I}^{+}\right) \frac{1}{\widetilde{\Delta}^{-}}=R,
\end{aligned}
$$

which implies $T \Phi \in \mathbb{X}_{0}$. Hence, $T\left(\mathbb{X}_{0}\right) \subset \mathbb{X}_{0}$.

Then, we prove that $T$ is a contracting mapping. In fact, for every $\Phi, \Psi \in \mathbb{X}_{0}$, we have

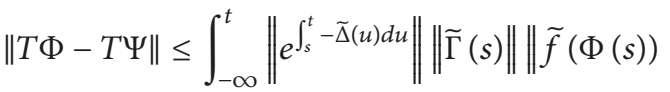

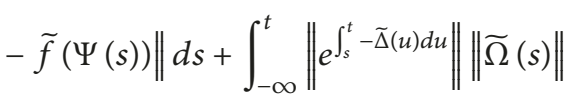

$$
\begin{aligned}
& \cdot\|\widetilde{g}(\Phi(s-\tau(s)))-\tilde{g}(\Psi(s-\tau(s)))\| d s \\
& \leq \int_{-\infty}^{t} e^{-\widetilde{\Delta}^{-}(t-s)} \widetilde{\Gamma}^{+}\|\tilde{f}(\Phi(s))-\tilde{f}(\Psi(s))\| d s \\
& +\int_{-\infty}^{t} e^{-\widetilde{\Delta}^{-}(t-s)} \widetilde{\Omega}^{+} \| \widetilde{g}(\Phi(s-\tau(s))) \\
& -\tilde{g}(\Psi(s-\tau(s))) \| d s \\
& \leq \widetilde{\Gamma}^{+} L_{f} \sum_{i=1}^{n} \sum_{B}\left|\Phi_{i}^{B}-\Psi_{i}^{B}\right| \int_{-\infty}^{t} e^{-\widetilde{\Delta}^{-}(t-s)} d s \\
& +\widetilde{\Omega}^{+} L_{g} \sum_{i=1}^{n} \sum_{B}\left|\Phi_{i}^{B}-\Psi_{i}^{B}\right| \int_{-\infty}^{t} e^{-\widetilde{\Delta}^{-}(t-s)} d s \leq\left(\widetilde{\Gamma}^{+} L_{f}\right. \\
& \left.+\widetilde{\Omega}^{+} L_{g}\right) \frac{1}{\widetilde{\Delta}^{-}}\|\Phi-\Psi\| .
\end{aligned}
$$

Hence, $T: \mathbb{X}_{0} \longrightarrow \mathbb{X}_{0}$ is a contracting mapping. Therefore, by the Banach fixed point theorem, there exists a unique point 
$\Phi^{*} \in \mathbb{X}_{0}$ such that $T \Phi^{*}=\Phi^{*}$; that is, system (14) has a unique almost periodic solution. In view of Remark 4 , we know that system (8) has a unique almost periodic solution. The proof is complete.

\section{Almost Periodic Synchronization}

In this section, we will investigate the global asymptotic synchronization problem of Clifford-valued CNNs with discrete delays. To this end, we consider the system (8) as the drive system and design the response system as

$$
\begin{aligned}
\dot{y}_{i}(t)= & -\delta_{i}(t) y_{i}(t)+\sum_{j=1}^{n} \gamma_{i j}(t) f_{j}\left(y_{j}(t)\right) \\
& +\sum_{j=1}^{n} \omega_{i j}(t) g_{j}\left(y_{j}\left(t-\tau_{j}(t)\right)\right)+I_{i}(t) \\
& +U_{i}(t), \\
y_{i}(t)= & \rho_{i}(t), \quad t \in[-\tau, 0], i=1,2, \ldots, n,
\end{aligned}
$$

where $\rho_{i} \in C([-\tau, 0], \mathscr{A}), Y_{i}(t) \in \mathscr{A}$ represents the state of the response system, $U_{i}(t)$ is a state-feedback controller, and the rest notation is the same as those in system (8).

Let $z_{i}^{A}(t)=y_{i}^{A}(t)-x_{i}^{A}(t)$; then, from (8) and (29), we obtain the following error system:

$$
\begin{aligned}
& \dot{z}_{i}(t)=-\delta_{i}(t) z_{i}(t)+\sum_{j=1}^{n} \gamma_{i j}(t) \\
& \cdot\left(f_{j}\left(y_{j}(t)\right)-f_{j}\left(x_{j}(t)\right)\right)+\sum_{j=1}^{n} \omega_{i j}(t) \\
& \cdot\left[g_{j}\left(y_{j}\left(t-\tau_{j}(t)\right)\right)-\left(g_{j}\left(x_{j}\left(t-\tau_{j}(t)\right)\right)\right]\right. \\
& +U_{i}(t), \quad t>0, \\
& z_{i}(t)=\varrho_{i}(t), \quad t \in[-\tau, 0], \quad i=1,2, \ldots, n,
\end{aligned}
$$

where $\varrho_{i}(t)=\rho_{i}(t)-\varphi_{i}(t)$. To realize the global asymptotic synchronization of the drive-response system, we choose the following state-feedback controller

$$
U_{i}(t)=\theta_{i}(t) z_{i}(t)+\sum_{j=1}^{n} \pi_{i j}(t) h_{j}\left(z_{j}(t)\right)
$$

where $i=1,2, \ldots, n, \theta_{i}: \mathbb{R} \longrightarrow \mathbb{R}^{+}, \pi_{i j}: \mathbb{R} \longrightarrow \mathscr{A}, h_{j}:$ $\mathscr{A} \longrightarrow \mathscr{A}$. By using a similar approach of transforming system (8) into system (14) and adopting the similar notation there, system (30) can be transformed into the following real-valued system:

$$
\begin{aligned}
& \dot{z}_{i}^{A}(t)=-\delta_{i}(t) z_{i}^{A}(t)+\sum_{j=1}^{n} \sum_{B} \gamma_{i j}^{A \cdot \bar{B}}(t) \\
& \cdot\left(f_{j}^{B}\left(y_{j}(t)\right)-f_{j}^{B}\left(x_{j}(t)\right)\right) \\
& +\sum_{j=1}^{n} \sum_{B} \omega_{i j}^{A \cdot \bar{B}}(t) \\
& \cdot\left[g_{j}^{B}\left(y_{j}\left(t-\tau_{i j}(t)\right)\right)-\left(g_{j}^{B}\left(x_{j}\left(t-\tau_{i j}(t)\right)\right)\right)\right], \\
& +\theta_{i}(t) z_{i}^{A}(t)+\sum_{j=1}^{n} \sum_{B} \pi_{i j}^{A \cdot \bar{B}}(t) h_{j}^{B}\left(z_{j}(t)\right), \\
& z_{i}^{A}(t)=\varrho_{i}^{A}(t), \\
& t \in[-\tau, 0], t \in[-\tau, 0], i=1,2, \ldots, n .
\end{aligned}
$$

Remark 7. Under the premise that system (8) has an almost periodic solution, the synchronization of almost periodic system (8) and almost periodic system (29) is called the almost periodic synchronization.

Theorem 8. Let $\left(H_{1}\right)-\left(H_{3}\right)$ hold. Suppose the following.

$\left(H_{4}\right)$ For $i, j=1,2, \ldots, n, \theta_{i} \in A P(\mathbb{R}, \mathbb{R})$ and $\pi_{i j} \in$ $A P(\mathbb{R}, \mathscr{A})$.

$\left(H_{5}\right)$ For $i=1,2, \ldots, n, h_{i}$ satisfy the idea that there exist constants $L_{i}^{h}$ such that $\left\|h_{i}(x)-h_{i}(y)\right\|_{\mathscr{A}} \leq L_{i}^{h}\|x-y\|_{\mathscr{A}}$ for all $x, y \in \mathscr{A}$, and $h_{i}(\mathbf{0})=\mathbf{0}$.

$\left(H_{6}\right)$ For $t \in \mathbb{R}, i=1,2, \ldots, n, \tau_{i} \in C^{1}\left(\mathbb{R}, \mathbb{R}^{+}\right)$with 1 $\tau_{i}^{\prime}(t)>0$ and

$$
\begin{aligned}
\Theta_{i}:= & \delta_{i}^{-}-\widehat{\theta}_{i}-\sum_{A} \sum_{B} \sum_{j=1}^{n} \gamma_{j i}^{A \cdot \bar{B}} L_{i}^{f}-\sum_{A} \sum_{B} \sum_{j=1}^{n} \frac{\omega_{j i}^{A \cdot \bar{B}} L_{i}^{g}}{1-\widehat{\tau}_{i}^{\prime}} \\
& -\sum_{A} \sum_{B} \sum_{j=1}^{n} \pi_{j i}^{A \cdot \bar{B}} L_{i}^{h}>0,
\end{aligned}
$$

where

$$
\begin{aligned}
\widehat{\theta_{i}} & =\sup _{t \in \mathbb{R}} \theta_{i}(t), \\
\widehat{M} & =\sup _{t \in \mathbb{R}}\|\bar{M}(t)\|, \\
\widehat{\gamma}_{j i}^{A \cdot \bar{B}} & =\sup _{t \in \mathbb{R}}\left\|\gamma_{j i}^{A \cdot \bar{B}}(t)\right\|, \\
\widehat{\tau}_{i}^{\prime} & =\sup _{t \in \mathbb{R}} \tau_{i}^{\prime}(t), \\
\widehat{m}_{j i}^{A \cdot \bar{B}} & =\sup _{t \in \mathbb{R}}\left\|m_{j i}^{A \cdot \bar{B}}(t)\right\|, \\
\widehat{\omega}_{j i}^{A \cdot \bar{B}} & =\sup _{t \in \mathbb{R}}\left\|\omega_{j i}^{A \cdot \bar{B}}(t)\right\|,
\end{aligned}
$$




$$
\begin{aligned}
& \delta_{i}^{-}=\inf _{t \in \mathbb{R}} \delta_{i}(t), \\
& L_{h}=\max _{1 \leq i \leq n}\left\{L_{i}^{h}\right\} .
\end{aligned}
$$$$
V(t)=V_{1}(t)+V_{2}(t)
$$

where

Then the drive system (8) and the response system (29) implement global asymptotical almost periodic synchronization.

Proof. By Theorem 6, system (8) has an almost periodic solution. In order to show that system (8) and system (29) are globally asymptotically synchronized, we consider a Lyapunov function

$$
\begin{aligned}
V_{1}(t) & =\sum_{i=1}^{n} \sum_{A}\left|z_{i}^{A}(t)\right| \\
V_{2} & =\sum_{A} \sum_{B} \sum_{i=1}^{n} \sum_{j=1}^{n} \widehat{\omega}_{j i}^{A \cdot \bar{B}} \frac{L_{i}^{g}}{1-\widehat{\tau}_{i}^{\prime}} \int_{t-\tau_{i}(t)}^{t} \sum_{Q}\left|z_{i}^{Q}(s)\right| d s .
\end{aligned}
$$

Calculating the derivatives of $V_{1}$ and $V_{2}$ along the solutions of system (32), we have

$$
\begin{aligned}
D^{+} V_{1}(t)= & \sum_{i=1}^{n} \sum_{A} \dot{z}_{i}^{A}(t) \operatorname{sign}\left\{z_{i}^{A}(t)\right\} \\
\leq & -\sum_{i=1}^{n} \sum_{A} \delta_{i}(t)\left|z_{i}^{A}(t)\right|+\sum_{i=1}^{n} \sum_{A} \sum_{B} \sum_{j=1}^{n}\left|\gamma_{i j}^{A \cdot \bar{B}}(t)\right|\left|f_{j}^{B}\left(y_{j}(t)\right)-f_{j}^{B}\left(x_{j}(t)\right)\right| \\
& +\sum_{i=1}^{n} \sum_{A} \sum_{B} \sum_{j=1}^{n}\left|\omega_{i j}^{A \cdot \bar{B}}(t)\right| \mid g_{j}^{B}\left(y_{j}\left(t-\tau_{j}(t)\right)-\left(g_{j}^{B}\left(x_{j}\left(t-\tau_{j}(t)\right)\right)\left|+\sum_{i=1}^{n} \sum_{A} \theta_{i}(t)\right| z_{i}^{A}(t) \mid\right.\right. \\
& +\sum_{i=1}^{n} \sum_{A} \sum_{B} \sum_{j=1}^{n}\left|\pi_{i j}^{A \cdot \bar{B}}(t)\right|\left|h_{j}^{B}\left(z_{j}(t)\right)\right| \\
\leq & \sum_{i=1}^{n} \sum_{A}\left|z_{i}^{A}(t)\right|\left(-\delta_{i}^{-}+\widehat{\theta}_{i}\right)+\sum_{A} \sum_{B} \sum_{i=1}^{n} \sum_{j=1}^{n} \widehat{\gamma}_{j i}^{A \cdot \bar{B}} L_{i}^{f} \sum_{\mathrm{Q}}\left|z_{i}^{\mathrm{Q}}(t)\right|+\sum_{A} \sum_{B} \sum_{i=1}^{n} \sum_{j=1}^{n} \widehat{\omega}_{j i}^{A \cdot \bar{B}} L_{i}^{g} \sum_{\mathrm{Q}}\left|z_{i}^{\mathrm{Q}}\left(t-\tau_{i}(t)\right)\right| \\
& +\sum_{A} \sum_{B} \sum_{i=1}^{n} \sum_{j=1}^{n} \widehat{\pi}_{j i}^{A \cdot \bar{B}} L_{i}^{h} \sum_{\mathrm{Q}}\left|z_{i}^{\mathrm{Q}}(t)\right|
\end{aligned}
$$

and

$$
\begin{aligned}
& D^{+} V_{2}(t)=\sum_{A} \sum_{B} \sum_{i=1}^{n} \sum_{j=1}^{n} \frac{\widehat{\omega}_{j i}^{A \cdot \bar{B}} L_{i}^{g}}{1-\widehat{\tau}_{i}^{\prime}} \sum_{\mathrm{Q}}\left|z_{i}^{\mathrm{Q}}(t)\right| \\
& \quad-\sum_{A} \sum_{B} \sum_{i=1}^{n} \sum_{j=1}^{n} \frac{\widehat{\omega}_{j i}^{A \cdot \bar{B}} L_{i}^{g}\left(1-\tau_{i}^{\prime}(t)\right)}{1-\widehat{\tau}_{i}^{\prime}} \\
& \cdot \sum_{\mathrm{Q}}\left|z_{i}^{\mathrm{Q}}\left(t-\tau_{i}(t)\right)\right| \\
& \leq \sum_{A} \sum_{B} \sum_{i=1}^{n} \sum_{j=1}^{n} \frac{\widehat{\omega}_{j i}^{A \cdot \bar{B}} L_{i}^{g}}{1-\widehat{\tau}_{i}^{\prime}} \sum_{\mathrm{Q}}\left|z_{i}^{\mathrm{Q}}(t)\right| \\
& \quad-\sum_{A} \sum_{B} \sum_{i=1}^{n} \sum_{j=1}^{n} \widehat{\omega}_{j i}^{A \cdot \bar{B}} L_{i}^{g} \sum_{\mathrm{Q}}\left|z_{i}^{\mathrm{Q}}\left(t-\tau_{i}(t)\right)\right| .
\end{aligned}
$$

Hence,

$$
\begin{aligned}
& \cdot \sum_{Q}\left|z_{i}^{\mathrm{Q}}(t)\right|+\sum_{A} \sum_{B} \sum_{i=1}^{n} \sum_{j=1}^{n} \widehat{\pi}_{j i}^{A \cdot \bar{B}} L_{i}^{h} \sum_{\mathrm{Q}}\left|z_{i}^{\mathrm{Q}}(t)\right| \\
& =\sum_{i=1}^{n} \sum_{\mathrm{Q}}\left|z_{i}^{\mathrm{Q}}(t)\right|\left[-\delta_{i}^{-}+\widehat{\theta}_{i}+\sum_{A} \sum_{B} \sum_{j=1}^{n} \widehat{\gamma}_{j i}^{A \cdot \bar{B}} L_{i}^{f}\right. \\
& \left.+\sum_{A} \sum_{B} \sum_{j=1}^{n} \frac{\omega_{j i}^{A \cdot \bar{B}} L_{i}^{g}}{1-\widehat{\tau}_{i}^{\prime}}+\sum_{A} \sum_{B} \sum_{j=1}^{n} \hat{\pi}_{j i}^{A \cdot \bar{B}} L_{i}^{h}\right] \leq-V_{1}(t) \\
& \cdot \min _{1 \leq i \leq n}\left\{\Theta_{i}\right\} .
\end{aligned}
$$

Integrating the above inequality over interval $\left[t_{0}, t\right]$, we get

$$
\begin{aligned}
D^{+} V(t) & =D^{+} V_{1}(t)+D^{+} V_{2}(t) \leq \sum_{i=1}^{n} \sum_{A}\left|z_{i}^{A}(t)\right|\left(-\delta_{i}^{-}\right. \\
\left.+\widehat{\theta}_{i}\right) & +\sum_{A} \sum_{B} \sum_{i=1}^{n} \sum_{j=1}^{n}\left(\widehat{\gamma}_{j i}^{A \cdot \bar{B}} L_{i}^{f}+\frac{\widehat{\omega}_{j i}^{A \cdot \bar{B}} L_{i}^{g}}{1-\widehat{\tau}_{i}^{\prime}}\right)
\end{aligned}
$$$$
V(t)+\min _{1 \leq i \leq n}\left\{\Theta_{i}\right\} \int_{t_{0}}^{t} V_{1}(s) d s \leq V\left(t_{0}\right)<+\infty,
$$ 
Thus,

$$
\begin{aligned}
\lim \sup _{t \rightarrow+\infty} \int_{t_{0}}^{t} V_{1}(s) d s \leq V\left(t_{0}\right) \leq \frac{V\left(t_{0}\right)}{\min _{1 \leq i \leq n}\left\{\Theta_{i}\right\}} \\
\quad<+\infty .
\end{aligned}
$$

Therefore, $\lim _{t \rightarrow+\infty} V_{1}(t)=0$. By the definition of $V_{1}(t)$, we obtain that $\lim _{t \rightarrow+\infty}\left|y_{i}^{B}(t)-x_{i}^{B}(t)\right|=0$, for all $B \in \Lambda$. Hence, the drive system (8) and the response system (29) are globally asymptotically synchronized. The proof is complete.

\section{A Numerical Example}

In this section, we present an example to illustrate our results.
Example 1. For $m=2, n=2$, take the following Cliffordvalued $\mathrm{CNN}$ as the drive system:

$$
\begin{aligned}
\dot{x}(t)= & -\Delta(t) x(t)+\Gamma(t) f(x(t)) \\
& +\Omega(t) g(x(t-\tau(t)))+I(t), \quad t>0, \\
x(t)= & \varphi(t), \quad t \in[-\tau, 0]
\end{aligned}
$$

and the response system is designed as

$$
\begin{aligned}
& \dot{y}(t)=-\Delta(t) y(t)+\Gamma(t) f(y(t)) \\
& +\Omega(t) g(y(t-\tau(t)))+I(t)+U(t), \\
& t>0 \text {, } \\
& x(t)=\phi(t), \quad t \in[-\tau, 0],
\end{aligned}
$$

where

$$
\begin{aligned}
& \Delta(t)=\left(\begin{array}{cc}
2+\sin \sqrt{2} t & 0 \\
0 & 4+\sin t
\end{array}\right) \\
& \Gamma(t)=\left(\begin{array}{cc}
0.2 \sin t+\sin t e_{1} & 0.1+0.3 \cos t e_{2}+0.6 e_{12} \\
0.05-0.2 \cos \sqrt{5} t e_{2}+0.4 e_{12} & 0.1+0.2 e_{1}+0.05 \sin \sqrt{3} t e_{12}
\end{array}\right), \\
& \Omega(t)=\left(\begin{array}{cc}
0.3 \sin \sqrt{3} t+0.01 \sin t e_{1} & 0.1+0.02 \cos \sqrt{3} t e_{2}+0.3 e_{12} \\
0.05-0.2 \sin t e_{2}+0.05 e_{12} & 0.2+0.2 e_{1}+0.05 \sin \sqrt{3} t e_{12}
\end{array}\right) \\
& f_{j}\left(x_{j}\right)=\frac{1}{65} \sin x_{j}^{0}-\frac{1}{70} \sin \sqrt{2} x_{j}^{2} e_{2}, \\
& g_{j}\left(x_{j}\right)=\frac{1}{58} \cos \sqrt{3} x_{j}^{2} e_{2}-\frac{1}{55} \sin \sqrt{6} x_{j}^{12} e_{12}, \\
& U(t)=\theta_{i}(t) e(t)+\Pi(t) h(z(t-\sigma(t))), \\
& h_{j}\left(z_{j}\right)=\frac{1}{75} \sin z_{j}^{0}-\frac{1}{68} \sin \sqrt{2} z_{j}^{2} e_{2}, \\
& \theta(t)=\left(\begin{array}{cc}
0.01+0.03 \sin 2 t & 0 \\
0 & 0.1+0.05 \cos \sqrt{2} t
\end{array}\right) \\
& \left(\begin{array}{l}
I_{1}(t) \\
I_{2}(t)
\end{array}\right)=\left(\begin{array}{c}
\frac{1}{10} \sin x_{1}^{0}(t)+\frac{1}{15} \sin \sqrt{3} x_{1}^{12}(t) e_{12} \\
\frac{1}{10} \sin x_{2}^{0}(t)+\frac{1}{15} \sin \sqrt{3} x_{2}^{12}(t) e_{12}
\end{array}\right) \\
& \left(\begin{array}{c}
\tau_{1}(t) \\
\tau_{2}(t)
\end{array}\right)=\left(\begin{array}{c}
0.1 \sin 3.2 t+0.2 \\
0.1 \sin 3.2 t+0.3
\end{array}\right) \\
& \Pi(t)=\left(\begin{array}{cc}
0.2 \sin \sqrt{2} t+0.01 \sin t e_{1} & 0.1+0.3 \cos t e_{2}+0.6 e_{12} \\
0.05-0.2 \cos \sqrt{5} t e_{2}+0.3 e_{12} & 0.1+0.2 e_{1}+0.05 \sin \sqrt{3} t e_{12}
\end{array}\right) .
\end{aligned}
$$


According to their definitions, we have

$$
\begin{aligned}
& \Gamma^{0}(t)=\left(\begin{array}{cc}
0.2 \sin t & 0.1 \\
0.05 & 0.1
\end{array}\right), \\
& \Gamma^{1}(t)=\left(\begin{array}{cc}
\sin t & 0 \\
0 & 0.2
\end{array}\right) \\
& \Gamma^{2}(t)=\left(\begin{array}{cc}
0 & 0.3 \cos t \\
-0.2 \cos \sqrt{5} t & 0
\end{array}\right), \\
& \Gamma^{12}(t)=\left(\begin{array}{cc}
0 & 0.6 \\
0.4 & 0.05 \sin \sqrt{3} t
\end{array}\right), \\
& \Omega^{0}(t)=\left(\begin{array}{cc}
0.3 \sin \sqrt{3} t & 0.1 \\
0.05 & 0.2
\end{array}\right), \\
& \Omega^{1}(t)=\left(\begin{array}{cc}
0.01 \sin t & 0 \\
0 & 0.2
\end{array}\right), \\
& \Omega^{2}(t)=\left(\begin{array}{cc}
0 & 0.02 \cos \sqrt{3} t \\
-0.2 \sin t & 0
\end{array}\right), \\
& \Omega^{12}(t)=\left(\begin{array}{cc}
0 & 0.3 \\
0.05 & 0.05 \sin \sqrt{3} t
\end{array}\right), \\
& \Pi^{0}(t)=\left(\begin{array}{cc}
0.2 \sin \sqrt{2} t & 0.1 \\
0.05 & 0.1
\end{array}\right), \\
& \Pi^{1}(t)=\left(\begin{array}{cc}
0.01 \sin t & 0 \\
0 & 0.2
\end{array}\right), \\
& \Pi^{2}(t)=\left(\begin{array}{cc}
0 & 0.3 \cos t \\
-0.2 \cos \sqrt{5} t & 0
\end{array}\right), \\
& \Pi^{12}(t)=\left(\begin{array}{cc}
0 & 0.6 \\
0.3 & 0.05 \sin \sqrt{3} t
\end{array}\right),
\end{aligned}
$$

and

$$
\begin{aligned}
\widetilde{\Gamma} & =\left(\begin{array}{cccc}
\Gamma^{0} & \Gamma^{\overline{1}} & \Gamma^{\overline{2}} & \Gamma^{\overline{12}} \\
\Gamma^{1} & \Gamma^{1 \cdot \overline{1}} & \Gamma^{1 \cdot \overline{2}} & \Gamma^{1 \cdot \overline{1} 2} \\
\Gamma^{2} & \Gamma^{2 \cdot \overline{1}} & \Gamma^{2 \cdot \overline{2}} & \Gamma^{2 \cdot \overline{1} 2} \\
\Gamma^{12} & \Gamma^{12 \cdot \overline{1}} & \Gamma^{12 \cdot \overline{2}} & \Gamma^{12 \cdot \overline{12}}
\end{array}\right) \\
& =\left(\begin{array}{cccc}
\Gamma^{0} & -\Gamma^{1} & -\Gamma^{2} & -\Gamma^{12} \\
\Gamma^{1} & \Gamma^{0} & -\Gamma^{12} & \Gamma^{2} \\
\Gamma^{2} & \Gamma^{12} & \Gamma^{0} & -\Gamma^{1} \\
\Gamma^{12} & -\Gamma^{2} & \Gamma^{1} & \Gamma^{0}
\end{array}\right),
\end{aligned}
$$

$$
\begin{aligned}
\widetilde{\Omega} & =\left(\begin{array}{cccc}
\Omega^{0} & \Omega^{\overline{1}} & \Omega^{\overline{2}} & \Omega^{\overline{12}} \\
\Omega^{1} & \Omega^{1 \cdot \overline{1}} & \Omega^{1 \cdot \overline{2}} & \Omega^{1 \cdot \overline{12}} \\
\Omega^{2} & \Omega^{2 \cdot \overline{1}} & \Omega^{2 \cdot \overline{2}} & \Omega^{2 \cdot \overline{12}} \\
\Omega^{12} & \Omega^{12 \cdot \overline{1}} & \Omega^{12 \cdot \overline{2}} & \Omega^{12 \cdot \overline{12}}
\end{array}\right) \\
& =\left(\begin{array}{cccc}
\Omega^{0} & -\Omega^{1} & -\Omega^{2} & -\Omega^{12} \\
\Omega^{1} & \Omega^{0} & -\Omega^{12} & \Omega^{2} \\
\Omega^{2} & \Omega^{12} & \Omega^{0} & -\Omega^{1} \\
\Omega^{12} & -\Omega^{2} & \Omega^{1} & \Omega^{0}
\end{array}\right), \\
\widetilde{\Pi} & =\left(\begin{array}{cccc}
\Pi^{0} & \Pi^{1} & \Pi^{2} & \Pi^{12} \\
\Pi^{1} & \Pi^{1 \cdot \overline{1}} & \Pi^{1 \cdot \overline{2}} & \Pi^{1 \cdot \overline{1}} \\
\Pi^{2} & \Pi^{2 \cdot \overline{1}} & \Pi^{2 \cdot \overline{2}} & \Pi^{2 \cdot \overline{1}} \\
\Pi^{12} & \Pi^{12 \cdot \overline{1}} & \Pi^{12 \cdot \overline{2}} & \Pi^{12 \cdot \overline{12}}
\end{array}\right) \\
& =\left(\begin{array}{cccc}
\Pi^{0} & -\Pi^{1} & -\Pi^{2} & -\Pi^{12} \\
\Pi^{1} & \Pi^{0} & -\Pi^{12} & \Pi^{2} \\
\Pi^{2} & \Pi^{12} & \Pi^{0} & -\Pi^{1} \\
\Pi^{12} & -\Pi^{2} & \Pi^{1} & \Pi^{0}
\end{array}\right) .
\end{aligned}
$$

By calculating, we obtain $\widetilde{\Delta}^{-}=1, \widetilde{\Gamma}^{+}=1.6, L_{1}^{f}=L_{2}^{f}=$ $1 / 65, L_{1}^{g}=L_{2}^{g}=1 / 55, \widetilde{\Omega}^{+}=0.75, L_{1}^{h}=L_{2}^{h}=1 / 68,\left(\gamma_{j i}^{A \cdot \bar{B}}\right)^{+}=$ 0.6, $\left(\omega_{j i}^{A \cdot \bar{B}}\right)^{+}=0.3,\left(\pi_{j i}^{A \cdot \bar{B}}\right)^{+}=0.6, \widehat{\theta_{1}}=0.04, \widehat{\theta_{2}}=0.15, c_{1}^{-}=1$, $c_{2}^{-}=3, \hat{\tau}_{i}^{\prime}=0.32, i, j=1,2$.

It is easy to get the following inequalities:

$$
\begin{aligned}
& \left(\widetilde{\Gamma}^{+} L_{f}+\widetilde{\Omega}^{+} L_{g}\right) \frac{1}{\widetilde{\Delta}^{-}}<1 \\
& \min _{1 \leq i \leq 2}\left\{-\delta_{i}^{-}+\widehat{\theta_{i}}+\sum_{A} \sum_{B} \sum_{j=1}^{2}\left(\gamma_{j i}^{A \cdot \bar{B}}\right)^{+} L_{i}^{f}\right. \\
& \left.+\sum_{A} \sum_{B} \sum_{j=1}^{2} \frac{\left(\omega_{j i}^{A \cdot \bar{B}}\right)^{+} L_{i}^{g}}{1-\widehat{\tau}_{i}^{\prime}}+\sum_{A} \sum_{B} \sum_{j=1}^{2}\left(\pi_{j i}^{A \cdot \bar{B}}\right)^{+} L_{i}^{h}\right\} \\
& \approx-0.015<0 .
\end{aligned}
$$

Therefore, all the conditions of Theorem 8 are satisfied. Hence, by Theorem 8 , the drive system (42) and the response system (43) implement global asymptotic almost periodic synchronization (see Figures 1-5).

\section{Conclusion}

In this paper, we studied the problem of the global asymptotic almost periodic synchronization for a class of Clifford-valued CNNs with discrete delays by decomposing the considered 

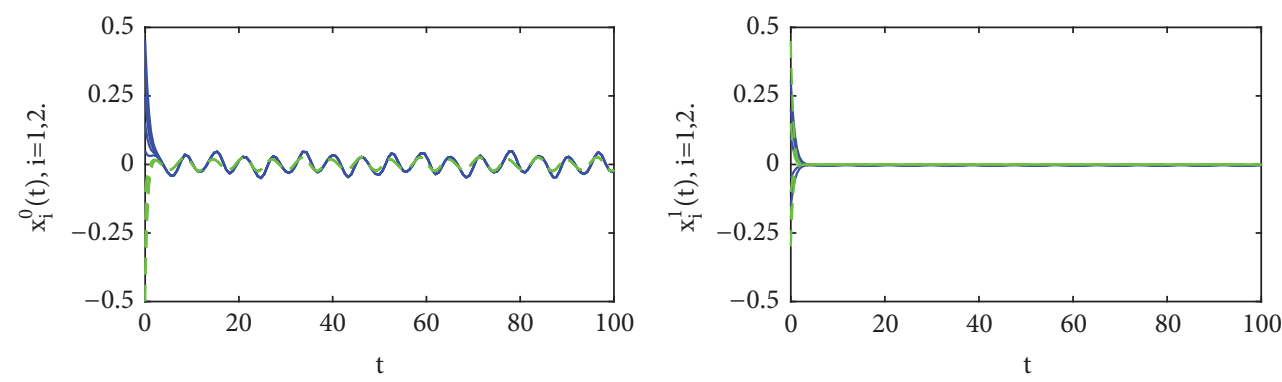

$-\mathrm{x}_{1}^{0}(\mathrm{t})$

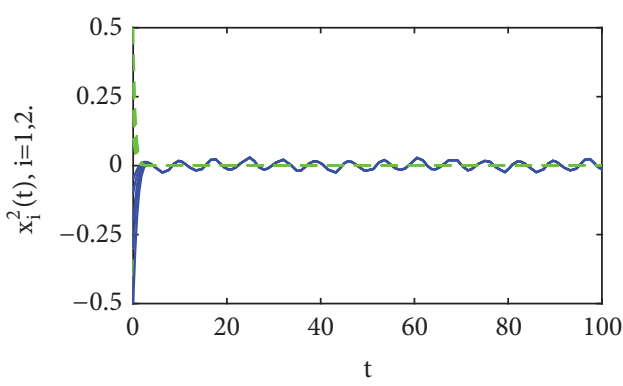

$-\mathrm{x}_{1}^{2}(\mathrm{t})$

$--\mathrm{x}_{2}^{2}(\mathrm{t})$ $-x_{1}^{1}(t)$

$--x_{2}^{1}(t)$

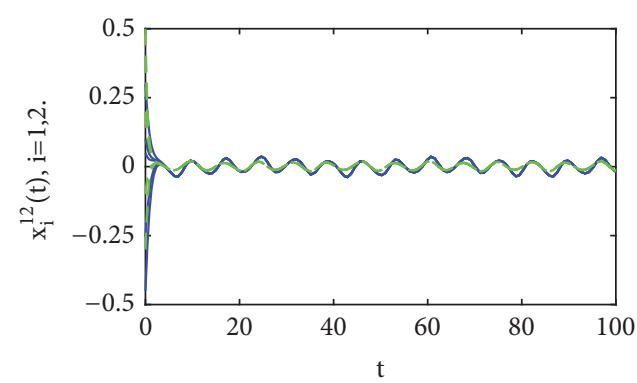

$-\mathrm{x}_{1}^{12}(\mathrm{t})$

$--x_{2}^{12}(t)$

FIgURE 1: The states of $x^{0}(t), x^{1}(t), x^{2}(t)$, and $x^{12}(t)$ in (42) without any controller.
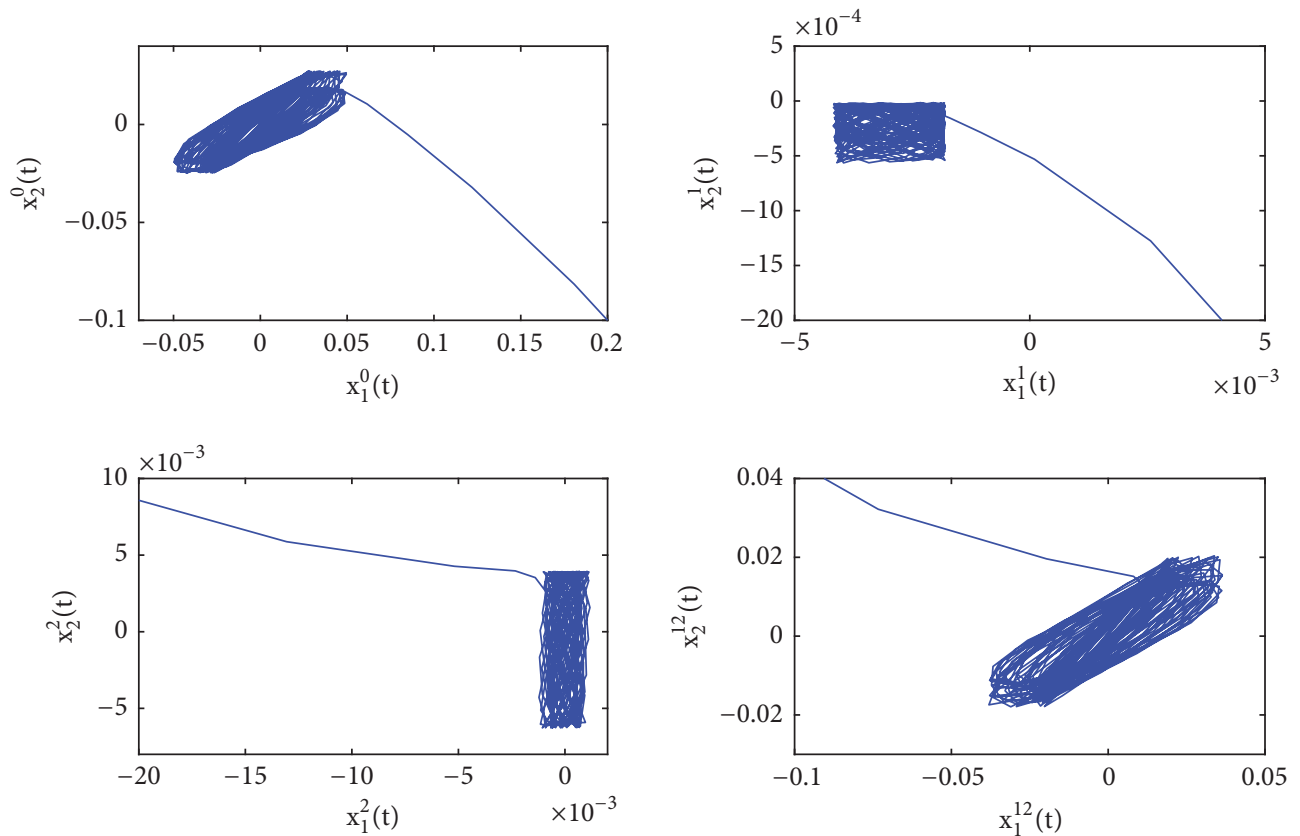

Figure 2: The states of four parts of $x_{1}(t)$ and $x_{2}(t)$ in 2-dimensional space without control. 

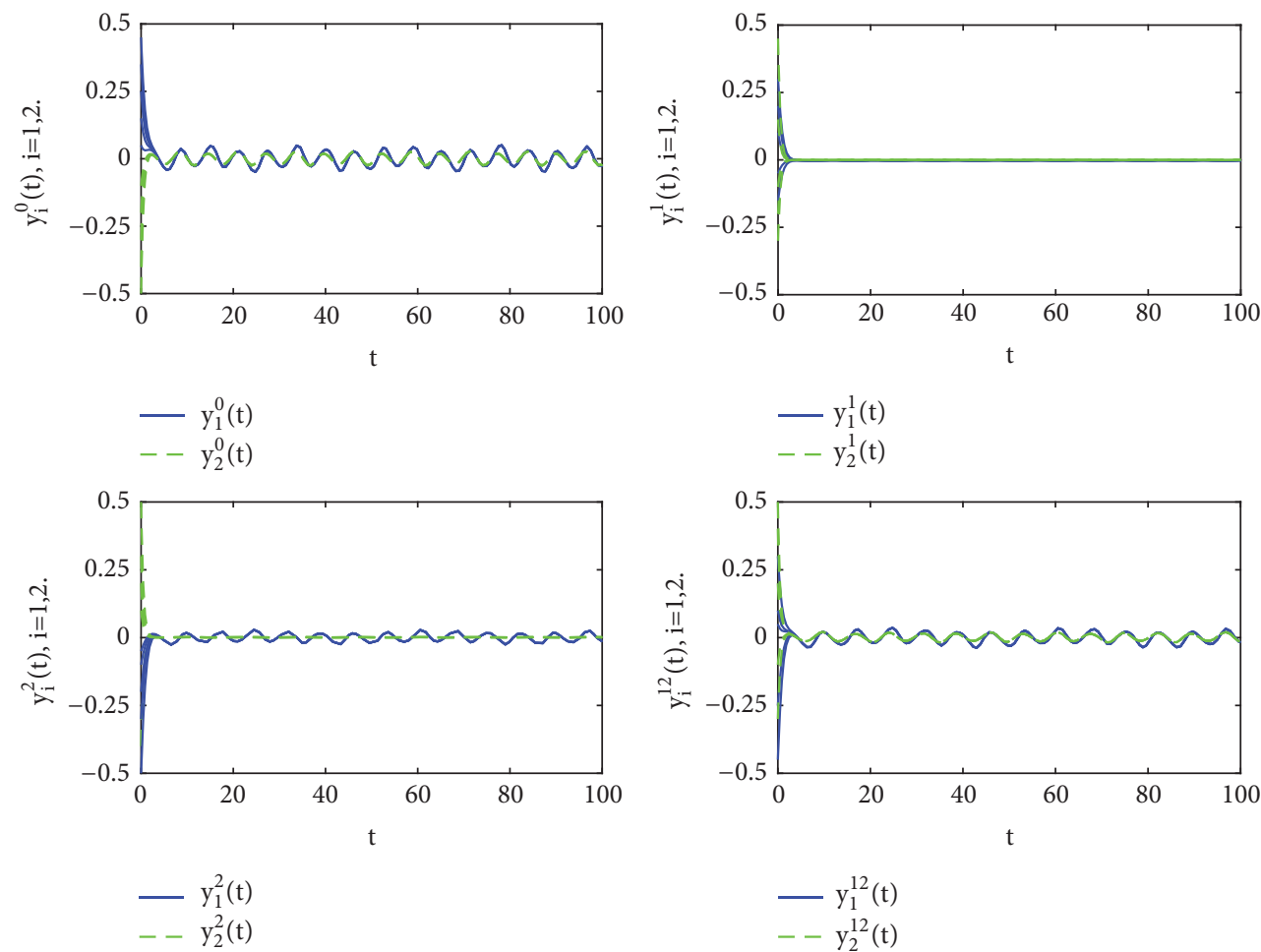

$$
\begin{aligned}
& -y_{1}^{12}(t) \\
& --y_{2}^{12}(t)
\end{aligned}
$$

FIgure 3: The states of $y^{0}(t), y^{1}(t), y^{2}(t)$, and $y^{12}(t)$ in (43) under controller $U(t)$.
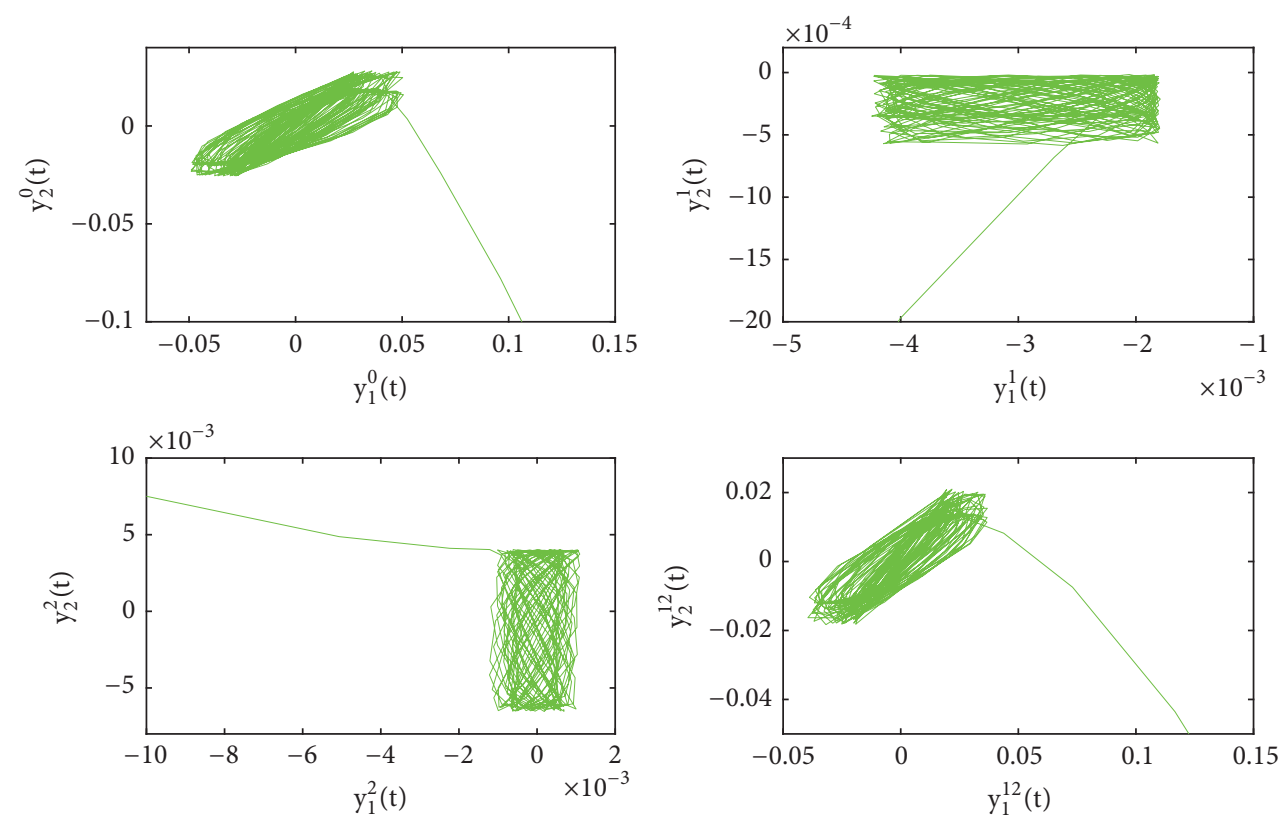

FIGURE 4: The states of four parts of $y_{1}(t)$ and $y_{2}(t)$ in 2-dimensional space under controller $U(t)$. 


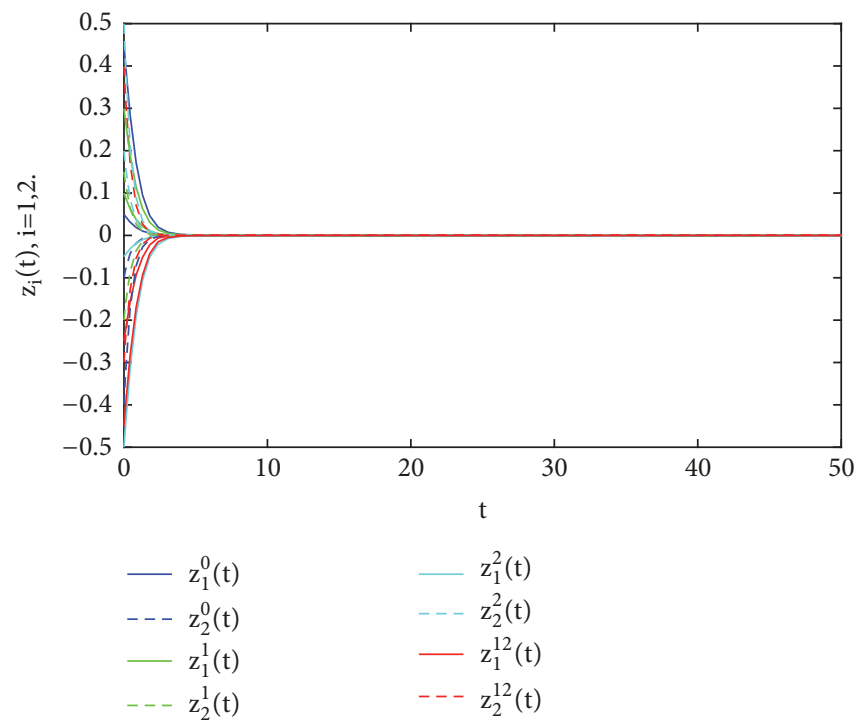

FIGURE 5: Synchronization errors $z(t)=y(t)-x(t)$.

neural networks into $2^{m^{2}} n$ real-valued systems and by designing a new state-feedback controller. Our results are new and our methods can be used to study other types of Cliffordvalued neural networks.

\section{Data Availability}

No data were used to support this study.

\section{Conflicts of Interest}

The authors declare that they have no conflicts of interest.

\section{Acknowledgments}

This work is supported by the National Natural Sciences Foundation of China under Grant No. 11861072.

\section{References}

[1] E. Hitzer, T. Nitta, and Y. Kuroe, "Applications of Clifford's geometric algebra," Advances in Applied Clifford Algebras (AACA), vol. 23, no. 2, pp. 377-404, 2013.

[2] S. Buchholz and G. Sommer, "On Clifford neurons and Clifford multi-layer perceptrons," Neural Networks, vol. 21, no. 7, pp. 925-935, 2008.

[3] E. Bayro-Corrochano, R. Vallejo, and N. Arana-Daniel, "Geometric preprocessing, geometric feedforward neural networks and Clifford support vector machines for visual learning," Neurocomputing, vol. 67, no. 1-4, pp. 54-105, 2005.

[4] J. Zhu and J. Sun, "Global exponential stability of Cliffordvalued recurrent neural networks," Neurocomputing, vol. 173, pp. 685-689, 2016.

[5] Y. Li and J. Xiang, "Existence and global exponential stability of anti-periodic solution for Clifford-valued inertial Cohen-Grossberg neural networks with delays," Neurocomputing, vol. 332, pp. 259-269, 2019.
[6] S. Tyagi, S. Abbas, and M. Kirane, "Global asymptotic and exponential synchronization of ring neural network with reaction-diffusion term and unbounded delay," Neural Computing and Applications, vol. 30, no. 2, pp. 487-501, 2018.

[7] H. Zhang, X. Y. Wang, and X. H. Lin, "Synchronization of complex-valued nerual network with sliding mode control," Journal of The Franklin Institute, vol. 353, no. 2, pp. 345-358, 2016.

[8] J. Gao, P. Zhu, W. Xiong, J. Cao, and L. Zhang, "Asymptotic synchronization for stochastic memristor-based neural networks with noise disturbance," Journal of The Franklin Institute, vol. 353, no. 13, pp. 3271-3289, 2016.

[9] Y. Li, B. Li, S. Yao, and L. Xiong, "The global exponential pseudo almost periodic synchronization of quaternion-valued cellular neural networks with time-varying delays," Neurocomputing, vol. 303, pp. 75-87, 2018.

[10] X. Yang, Q. Song, J. Cao, and J. Lu, "Synchronization of coupled markovian reaction-diffusion neural networks with proportional delays via quantized control," IEEE Transactions on Neural Networks and Learning Systems, vol. 30, no. 3, pp. 951958, 2019.

[11] C.-D. Zheng and Y. Xian, "On synchronization for chaotic memristor-based neural networks with time-varying delays," Neurocomputing, vol. 216, pp. 570-586, 2016.

[12] T. Hu, X. Zhang, and S. Zhong, "Global asymptotic synchronization of nonidentical fractional-order neural networks," Neurocomputing, vol. 313, pp. 39-46, 2018.

[13] S. Lakshmanan, M. Prakash, C. P. Lim, R. Rakkiyappan, P. Balasubramaniam, and S. Nahavandi, "Synchronization of an inertial neural network with time-varying delays and its application to secure communication," IEEE Transactions on Neural Networks and Learning Systems, vol. 29, no. 1, pp. 195207, 2018.

[14] R. Tang, X. Yang, and X. Wan, "Finite-time cluster synchronization for a class of fuzzy cellular neural networks via nonchattering quantized controllers," Neural Networks, vol. 113, pp. 79-90, 2019. 
[15] Y. Li, X. Meng, and Y. Ye, "Almost periodic synchronization for quaternion-valued neural networks with time-varying delays," Complexity, vol. 2018, Article ID 6504590, 13 pages, 2018.

[16] A. M. Fink, Almost Periodic Differential Equations, Springer, New York, NY, USA, 1974. 


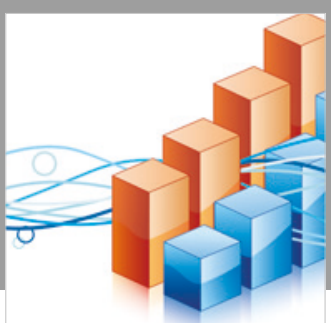

Advances in

Operations Research

\section{-n-m}
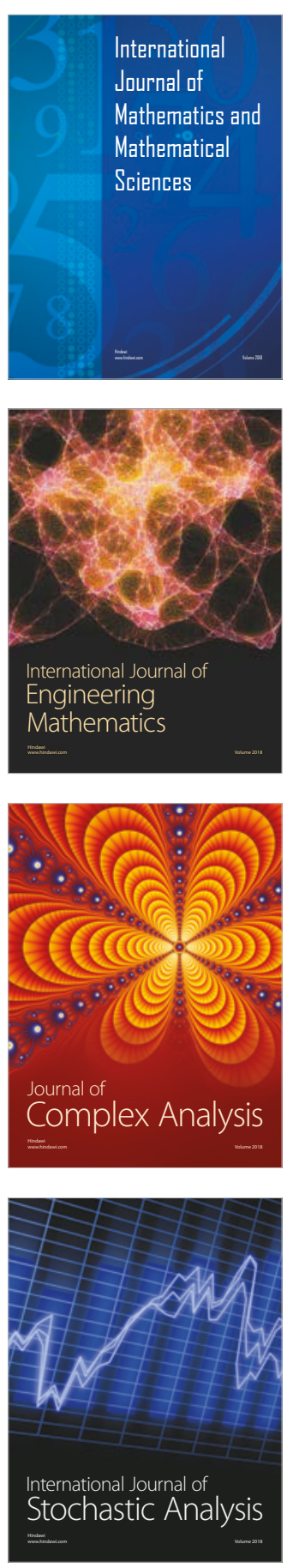
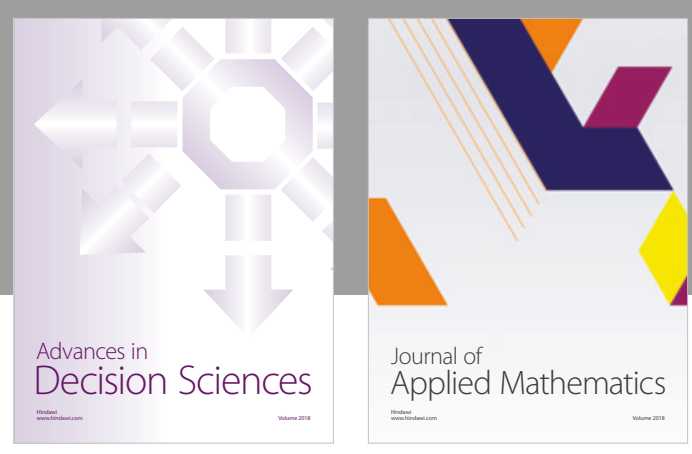

Journal of

Applied Mathematics
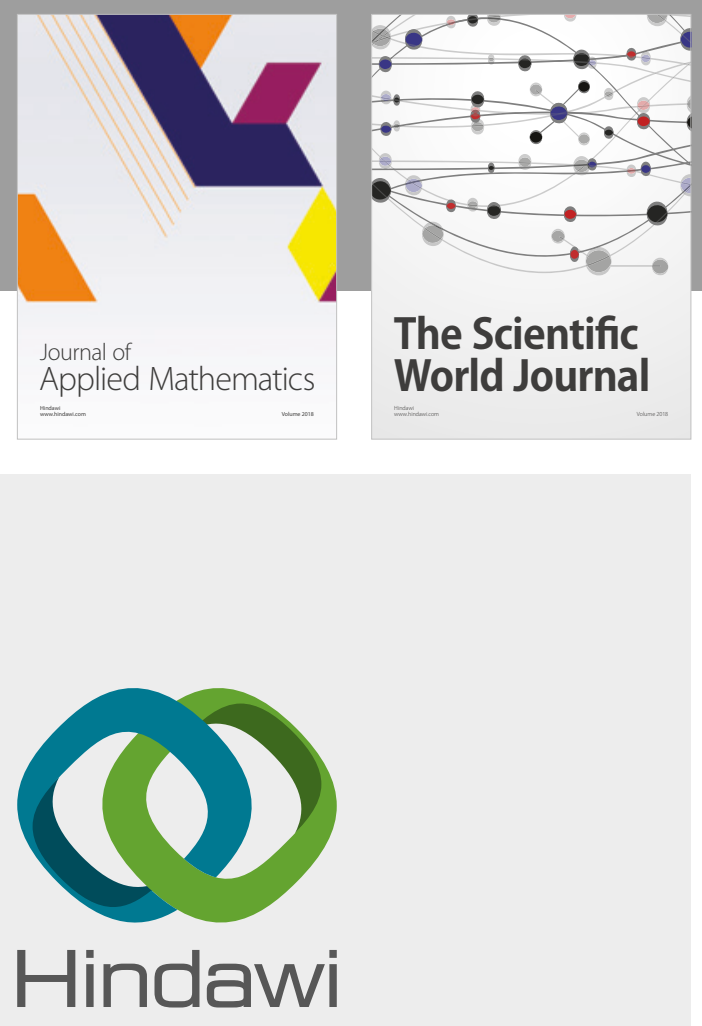

Submit your manuscripts at

www.hindawi.com

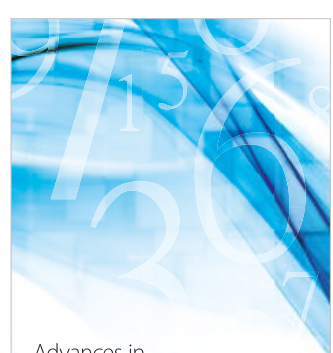

Advances in
Numerical Analysis
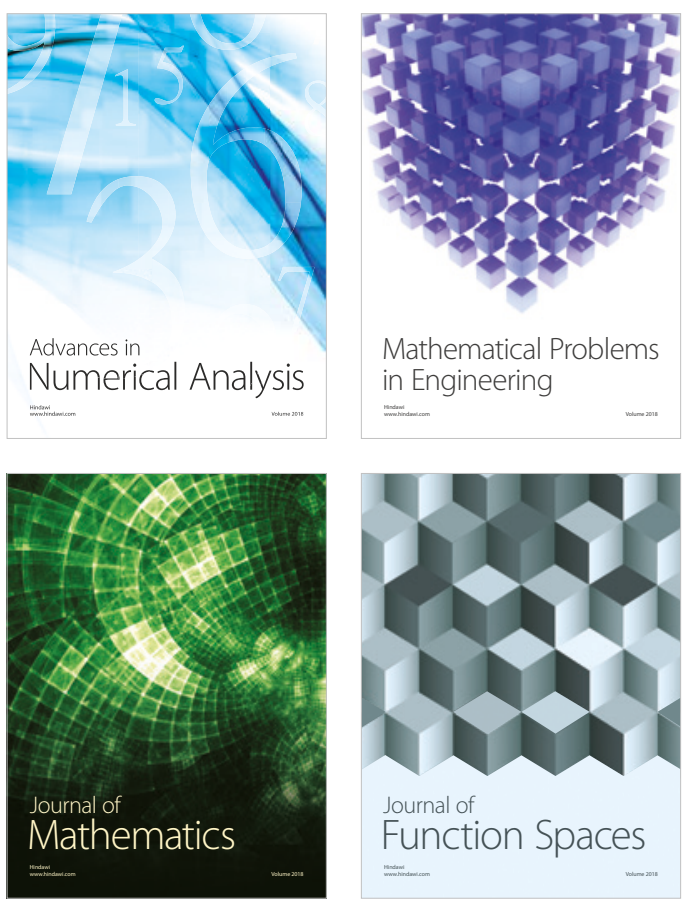

Mathematical Problems in Engineering

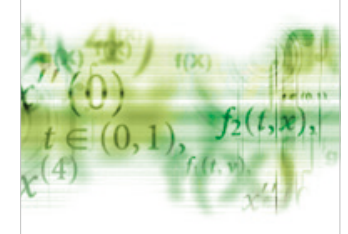

International Journal of

Differential Equations

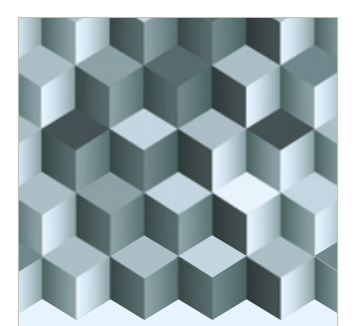

Journal of

Function Spaces
The Scientific

World Journal

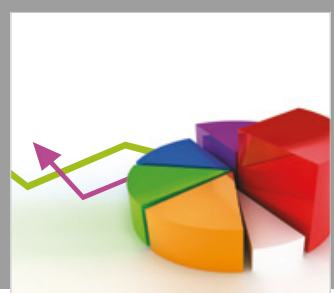

Journal of

Probability and Statistics
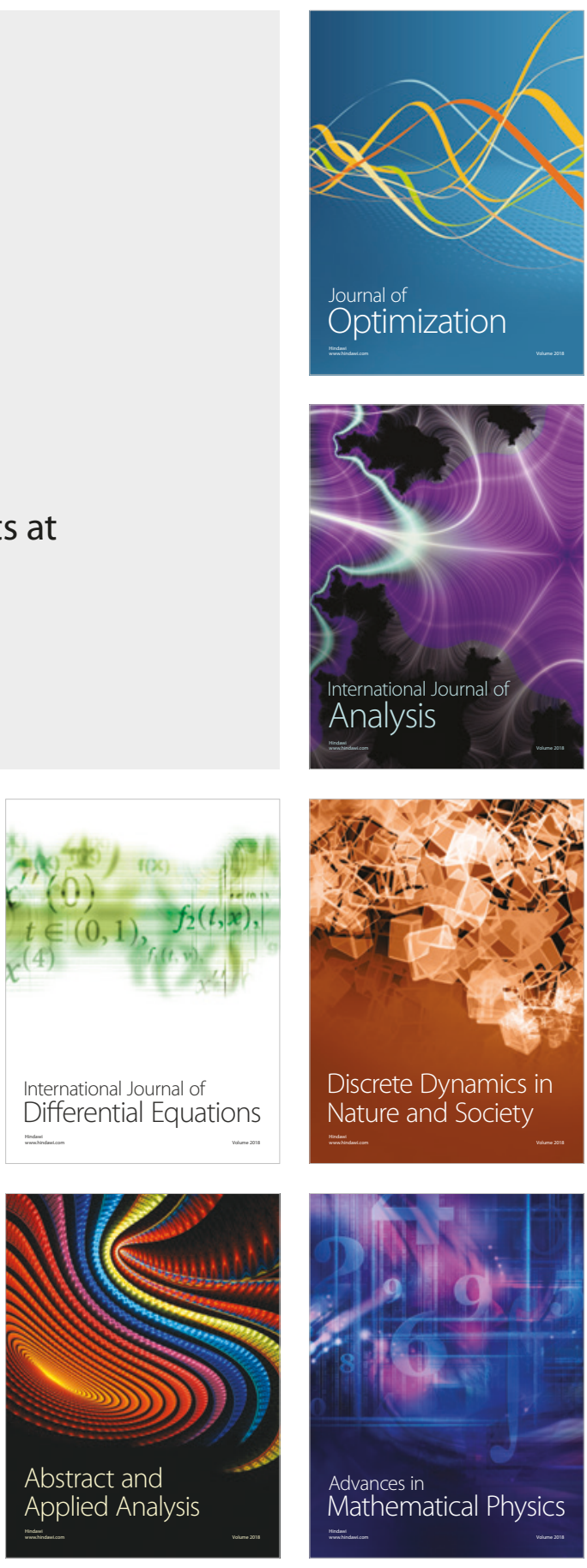